\title{
Corela
}

Cognition, représentation, langage

HS-18 | 2015

La reformulation : usages et contextes

\section{La reprise corrective dans les interactions en classe de français langue seconde}

\section{Evelyne Berger}

\section{(2) OpenEdition}

\section{Journals}

Édition électronique

URL : http://journals.openedition.org/corela/4170

DOI : $10.4000 /$ corela.4170

ISSN : $1638-573 \mathrm{X}$

\section{Éditeur}

Cercle linguistique du Centre et de I'Ouest - CerLICO

\section{Édition imprimée}

Date de publication : 15 novembre 2015

ISSN : 1638-5748

\section{Référence électronique}

Evelyne Berger, "La reprise corrective dans les interactions en classe de français langue seconde », Corela [En ligne], HS-18 | 2015, mis en ligne le 15 novembre 2015, consulté le 01 mai 2019. URL: http://journals.openedition.org/corela/4170 ; DOI : 10.4000/corela.4170

Ce document a été généré automatiquement le 1 mai 2019

\section{cc) (i)}

Corela - cognition, représentation, langage est mis à disposition selon les termes de la licence Creative Commons Attribution - Pas d'Utilisation Commerciale - Partage dans les Mêmes Conditions 4.0 International. 


\title{
La reprise corrective dans les interactions en classe de français langue seconde
}

\author{
Evelyne Berger
}

\section{Introduction}

1 La reprise, et la reformulation plus généralement, font l'objet d'une attention sans cesse renouvelée des chercheurs dans le domaine de l'apprentissage des langues secondes depuis de nombreuses années, tant ce procédé discursif est omniprésent dans les interactions exolingues. De nombreux travaux ont mis en évidence leur fonctionnement au sein de séquences accomplissant un travail linguistique : négociation de sens et de forme, étayage, correction/réparation d'un élément linguistique, etc. (cf. p.ex. Brouwer, 2004 ; De Pietro et al., 1989; Faraco, 2002 ; Hellermann, 2011; Hosoda, 2000; Krafft \& Dausendschön-Gay, 1993 ; Kurhila, 2006 ; Lyster \& Ranta, 1997 ; Vasseur, 2005).

2 Ainsi, la reprise, et notamment la reprise corrective, offre un potentiel acquisitionnel important pour les apprenants d'une langue seconde, dans la mesure où elle permet un travail sur les formes linguistiques. Au-delà de cette fonction métalinguistique, ce qui va nous intéresser cependant dans le cadre de cette étude sur la reprise corrective, c'est son fonctionnement interactionnel. Ainsi, nous examinerons des cas de reprise accomplissant une correction d'un propos antérieur, dans la mesure où le segment-source est modifié tout en poursuivant un projet communicatif (cf. 'embedded correction', Jefferson, 1987; voir aussi Brouwer et al., 2004 ; Fasel Lauzon \& Pekarek Doehler, 2013 ; Svennenig, 2004). Dans nos données, il apparaît que, bien au-delà de la résolution d'un problème linguistique, la reprise corrective est exploitée par le locuteur comme ressource pour se (ré)approprier la parole face à ses interlocuteurs.

3 Sur la base d'un corpus d'interactions en petits groupe en classe de français L2 et en mobilisant le cadre de l'analyse conversationnelle d'origine ethnométhodologique, le fonctionnement interactionnel de la reprise sera détaillé à partir d'extraits de 
conversation. Cette recherche espère ainsi enrichir notre compréhension du phénomène de la reprise en langue seconde en l'abordant à partir d'un angle interactionniste. La reprise se voit redéfinie dans cette perspective en tant que ressource discursive émergeant de la dynamique interactive et exploitée par le locuteur à toutes fins pratiques, au-delà de ses caractéristiques formelles (cf. Pekarek Doehler \& PochonBerger, 2010). D'autre part, la reprise corrective par des locuteurs de L2 n'est plus seulement envisagée comme étant impliquée dans un travail métalinguistique, mais comme un moyen que les locuteurs emploient pour structurer leur discours, se coordonner avec autrui et gérer leurs relations interpersonnelles.

\section{Etat de la question}

\subsection{La notion de reprise}

4 L'objet qui nous intéresse ici se présente sous la forme d'un recyclage d'un segment discursif antérieur, préservant un parallélisme formel (au niveau lexical, grammatical) avec celui-ci tout en comprenant une variation localisée sur un élément linguistique (cf. parties en gras dans l'exemple suivant) :

Exemple 1

01 MIC: et des maquilles,

$02 \quad(0.5)$

03 LOR: >maquillages< pou:r pour la bouche pour les yeux.

5 Dans cet exemple, l'élément linguistique 'des maquilles' (1.1) est rétrospectivement traité par l'interlocuteur comme un élément problématique devant être réparé au moyen d'une reprise (1.3) (il est à noter que dans certains cas, l'élément qui est corrigé ne constitue pas à priori une forme non-standard); raison pour laquelle nous adopterons le terme de reprise corrective.

6 Les linguistes ne se sont pas toujours accordés sur les phénomènes qu'ils catégorisent de reprise, répétition, reformulation. La reprise est parfois utilisée en tant que terme générique englobant différentes formes d'un retour sur un discours antérieur (cf. p.ex. Vasseur, 2005). Chez d'autres, la reprise se limite exclusivement à la répétition à la lettre (cf. p.ex. Vion, 1992). Dans ce dernier cas, toute modification, même minimale, d'un segment antérieur constitue une reformulation. La reformulation à son tour est également utilisée comme terme générique chez certains auteurs (cf. p.ex. Rabatel, 2010). Le choix de telle ou telle catégorie descriptive se fonde généralement sur le degré d'équivalence formelle entre l'énoncé-source et l'énoncé-reformulateur, distinguant ainsi entre ce qui relève d'une répétition à l'identique et ce qui relève d'une reprise avec modification à envergure variable. Cependant, lorsque l'on resitue la reprise ou reformulation dans la temporalité de l'interaction, ces distinctions perdent de leur pertinence. En effet, une reprise ou reformulation n'est jamais une reproduction identique d'un dire antérieur ; du fait même qu'elle est re-prise ou re-formulation, c'est-à-dire une deuxième production, elle se constitue en nouvelle action à part entière (cf. Pekarek Doehler \& Pochon-Berger, 2010). C'est précisément ce caractère réitératif qui constitue l'intérêt de ce procédé : on fait autre chose à partir d'un dire antérieur (cf. Rabatel, 2010). Comme le souligne SalazarOrvig (2000: 86) : "Ainsi la reprise ne serait pas uniquement réutilisation mais renvoi à un 
énoncé antérieur, un pont jeté entre deux discours.». Ainsi donc, au-delà des catégories formelles perçues par l'analyste, nous utiliserons le terme de reprise pour souligner le lien de ressemblance entre deux segments discursifs établi séquentiellement par les locuteurs eux-mêmes.

\subsection{Reprise et activité corrective}

7 L'implication de la reprise dans les activités correctives a souvent été observée dans la recherche sur les interactions exolingues et l'acquisition des langues secondes. Les travaux sur le corrective feedback (p.ex. Lyster, 1998; Lyster \& Ranta, 1997) notamment ont mis en évidence la présence de reprises en différentes positions séquentielles au sein de séquences de travail sur les formes. Lorsqu'elle est produite par l'interlocuteur, la reprise sert d'outil (mais n'est pas le seul) pour isoler un objet problématique et attirer l'attention de l'apprenant là-dessus, par exemple au moyen d'une répétition avec intonation interrogative qui invite à une confirmation ou une modification de la part du premier locuteur. L'interlocuteur, toujours, peut aussi avoir recours à la reprise pour fournir une forme linguistique plus appropriée, qui vient 'annuler' la précédente produite par l'apprenant. Dans ce cadre, la reprise accomplit en soi la correction nécessaire. Enfin, après la correction d'autrui, l'apprenant peut recourir à la reprise pour ratifier une correction, voire intégrer la forme cible dans le système linguistique. En général, la reprise permet ainsi de négocier, évaluer, reformater des formes linguistiques en vue de leur appropriation. La reprise, et notamment la reprise corrective, offre ainsi un potentiel acquisitionnel important pour les apprenants d'une langue seconde (cf. 'séquences potentiellement acquisitionnelles', De Pietro et al., 1989).

8 La fonction métalinguistique de la reprise est illustrée dans l'exemple suivant où trois élèves, apprenants de français L2, discutent de l'organisation d'une fête d'anniversaire :

\section{Exemple 2 [Corpus WBS Tschu-TG1-gs]}

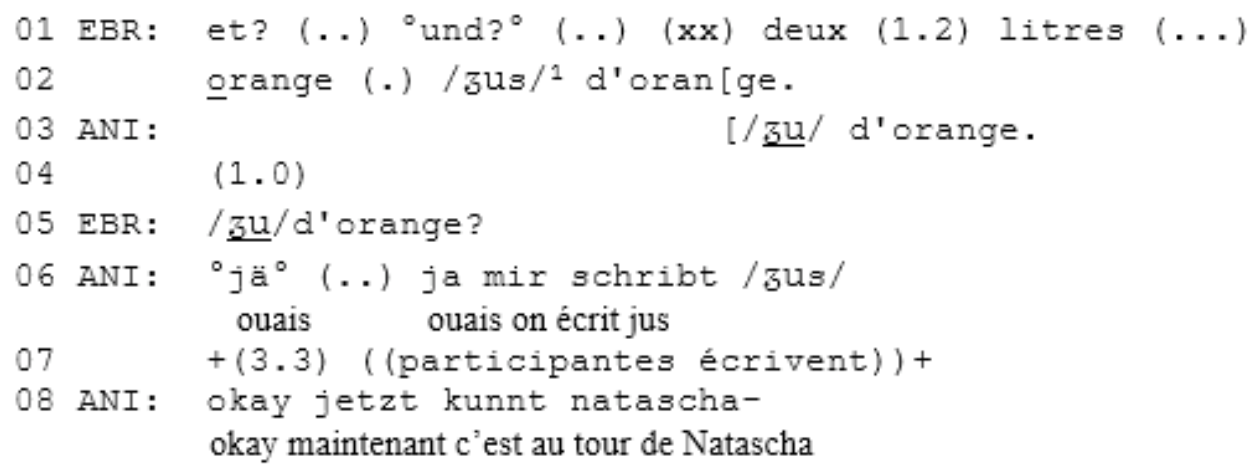

Dans cet extrait, un élément linguistique non-standard situé dans le tour de parole de l'une des élèves, Ebru, fait l'objet d'une correction par sa camarade Anila dans le tour subséquent ('/3u/ d'orange', 1.3). La correction est accomplie au moyen d'une reformulation de l'élément problématique en une version plus normative accompagné d'une accentuation prosodique. Cette correction est acceptée par Ebru, au moyen d'une répétition avec intonation interrogative invitant à une confirmation. Dans le tour suivant, Anila fournit la raison de sa correction, sous la forme d'un commentaire métalinguistique en L1. Ainsi, la séquence de correction est effectuée en trois pas: une forme problématique dans un premier tour de parole est identifiée et corrigée dans le second 
tour, puis ratifiée dans un troisième tour. L'activité de correction par l'interlocuteur implique la plupart du temps d'interrompre ou de suspendre le projet communicatif initial, afin de traiter le problème linguistique. Il y a donc momentanément une focalisation exercée sur le code linguistique (De Pietro et al., 1989), avant de poursuivre le projet communicatif antérieur, qui se manifeste dans cet extrait avec la continuation de la tâche (cf. 1.8).

10 Avec les travaux mentionnés plus haut, l'accent est mis sur la fonction de la reprise en tant que moyen d'appropriation des formes et structures linguistiques qui témoigne d'un effort de maintien de l'intercompréhension de la part des interactants, dont les moyens langagiers semblent déficients. Un nombre plus réduit d'études ont cependant identifié des fonctions interactives de la reprise ou reformulation en langue seconde: comme ressource pour légitimer la prise de parole (Pallotti, 2002; Vion \& Mittner, 1986) ou négocier des topics conversationnels (Faraco, 2002). C'est dans la lignée de ces travaux plus particulièrement que s'inscrit la présente étude.

\section{Cadre de l'étude}

\subsection{Les données}

11 Cette étude se base sur un corpus de 4h30min d'enregistrements audiovisuels d'interactions en classe de français langue seconde en Suisse alémanique. Il s'agit plus particulièrement de travaux en groupes se composant de 3-4 élèves accomplissant conjointement une tâche pédagogique dont l'objectif est de pratiquer l'oral en français. Il arrive parfois que ces élèves interagissent avec l'enseignante ou l'assistante de l'enseignante.

12 Les élèves sont des apprenants âgés de 13-14 ans qui se trouvent en $8^{\text {ème }}$ année scolaire au secondaire obligatoire inférieur (selon la numérotation du système scolaire suisse). Au moment des enregistrements, ils suivent pour la quatrième année consécutive un enseignement de français. Ce sont des locuteurs de français langue seconde de niveau intermédiaire.

13 Les enregistrements audiovisuels ont fait l'objet d'une transcription minutieuse rendant compte des détails de la production de la parole (p.ex. les chevauchements de tours de parole, mais aussi les aspects prosodiques tels que les faux-départs, l'intonation finale, des changements de débit ou de volume de voix sur certains segments; cf. conventions de transcription en annexe). Les segments de parole produits en suisse-allemand/allemand, L1 des élèves, font l'objet d'une traduction qui est donnée dans une autre police sous de la ligne de transcription principale. Les éléments linguistiques dont la prononciation est ambigüe sont transcrits phonétiquement d'après les conventions de l'Alphabet Phonétique International. Enfin, des phénomènes non-verbaux tels que les regards, certains gestes ou encore des mouvements corporels, sont également indiqués pour des moments spécifiques dans les extraits choisis. Ces phénomènes non-verbaux (ou: multimodaux) sont indiqués en italique sous la ligne de transcription principale.

\subsection{Procédures analytiques}

14 Dans la mesure où la reprise est un phénomène omniprésent dans les interactions en langues secondes (cf. Vasseur, 2005) - et en général d'ailleurs (cf. Tannen, 1989) -, une 
première étape analytique a consisté en la délimitation de l'objet d'étude. Les occurrences de reprise corrective ayant retenu notre attention partagent les traits séquentiels récurrents suivants :

- Source de la reprise. Il s'agit de reprises du discours d'autrui (hétéro-reprises) et de reprises entre élèves uniquement.

- Proximité de la reprise. Les reprises sont immédiates: l'énoncé-source et l'énoncéreformulateur sont séquentiellement adjacents.

- Environnement discursif. La reprise n'est pas isolée, mais intégrée à un tour de parole plus large accomplissant un projet communicatif différent de l'activité corrective.

- Positionnement séquentiel. La reprise se trouve en position initiale de l'énoncé-reformulateur.

Dans une deuxième étape, ces cas de reprise ont fait l'objet d'une analyse qualitative fine s'inscrivant dans l'approche de l'analyse conversationnelle d'origine éthnométhodologique. Les résultats de cette analyse sont présentés dans la suite de cet article au moyen d'exemples représentatifs. Les extraits seront décrits dans une perspective séquentielle (c.-à-d. prise en compte du déploiement temporel de la paroleen-interaction) et multimodale (c.-à-d. prise en compte des conduites verbales, prosodiques et non-verbales).

\subsection{Clarification terminologique}

Nous distinguons segment-source d'énoncé-source. Le segment-source représente le fragment de discours faisant l'objet subséquemment d'une reprise; il peut être de nature et de longueur variable, tel un mot, un syntagme, une partie d'énoncé ou encore un tour de parole entier. L'énoncé-source quant à lui correspond au tour de parole contenant le segment-source - et qui peut d'ailleurs se trouver avec celui-ci en parfaite équivalence. De la même façon, nous distinguons reprise et énoncé-reformulateur; ce dernier étant le tour de parole contenant la reprise (cf. Gülich \& Kotschi, 1987).

\section{Reprise et correction 'camouflée'}

Dans cette première partie d'analyse, nous nous intéresserons à la manière dont la reprise corrective est formatée sur le plan linguistique et prosodique. Nous montrerons que le formatage interactionnel particulier dont elle fait l'objet permet aux participants de procéder à la correction d'un élément linguistique de façon implicite et ainsi d'éviter de placer l'activité corrective au centre de l'attention.

Dans l'exemple 3 (qui constitue un extrait plus large de l'exemple 1), un groupe de trois élèves discute de possibles accessoires de mode qu'elles pourraient acheter pour aller à une fête, et ceci alors que l'assistante de l'enseignante se trouve près d'elles et les observe. Lorena produit une reprise corrective à la ligne 10, dont l'énoncé-source se trouve à la ligne 8 : 
Exemple 3 [Corpus WBS Tschu-TG1-sadj]

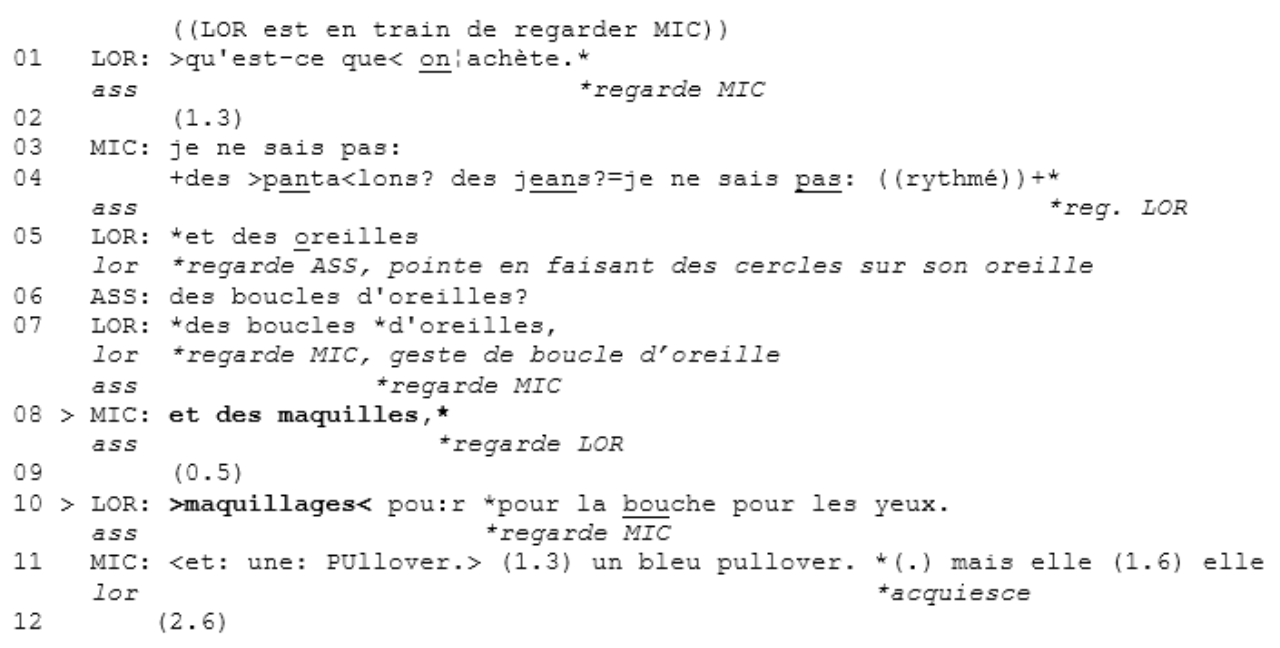

19 Deux élèves, Lorena et Michelle, énumèrent conjointement les différents accessoires, donnant ainsi lieu à une liste : Michelle propose 'des pantalons' (1. 4), suivie de Lorena qui propose 'des boucles d'oreilles' (qui fait l'objet d'une aide lexicale de la part de l'assistante, 1. 6-7). Michelle y ajoute un troisième accessoire : 'et des maquilles' (1. 8). Ce troisième élément présente une forme non-standard 'maquilles', qui n'est cependant pas traité comme problématique par la locutrice : il est produit sans hésitations et aucune demande d'aide n'est formulée. Bien au contraire, Michelle s'oriente vers la poursuite de la liste de la part de sa camarade, comme semble l'indiquer l'intonation continuative à la fin de son tour et son regard vers son interlocutrice.

20 Après une pause de 0.5 seconde, Lorena enchaîne par le biais d'une reprise corrective de l'élément problématique (à noter que seule la forme lexicale est reprise, sans le déterminant) tout en élaborant les contenus : 'maquillages pour la bouche pour les yeux' (1. 10). De manière intéressante, sur le plan prosodique, cet élément lexical est produit avec un débit accéléré et sans accentuation qui attirerait l'attention sur l'activité de correction. La reprise corrective n'est ainsi pas mise en évidence par un procédé prosodique ou formel. D'ailleurs, elle est intégrée au développement discursif subséquent: en effet, elle est suivie directement par la préposition 'pour', laquelle indique d'emblée une continuation syntaxique du tour de parole et exclut ainsi la possibilité d'une reprise corrective isolée.

21 En somme, hormis la pause de 0.5 seconde à la ligne 9 qui pourrait être l'indice d'un problème vers lequel Lorena semble s'orienter (dans la mesure où les changements de locuteur se font sans pause inter-tour pour les tours précédents), rien n'indique clairement une focalisation des participants sur le problème linguistique. Celui-ci est traité de manière implicite, voire 'camouflée', par Lorena. Ceci permet de poursuivre l'échange et d'avancer dans l'exécution de la tâche. Dans le tour suivant, Michelle ne ratifie d'ailleurs pas la reprise corrective ; bien au contraire, elle poursuit simplement la liste en proposant un quatrième accessoire, ce qui contribue à clore la liste.

Prenons un deuxième exemple. Il s'agit ici d'un autre groupe de filles qui discutent cette fois-ci d'un itinéraire possible entre deux endroits se nommant Marktplatz et Münsterplatz. Elles ont à disposition un plan de ville sur lequel elles élaborent leur itinéraire. Michelle est celle qui mène la description, avec l'aide ponctuelle de ses camarades Ebru et Serife. A 
la ligne 13, Serife enchaîne sur la description de Michelle au moyen d'une reprise corrective :

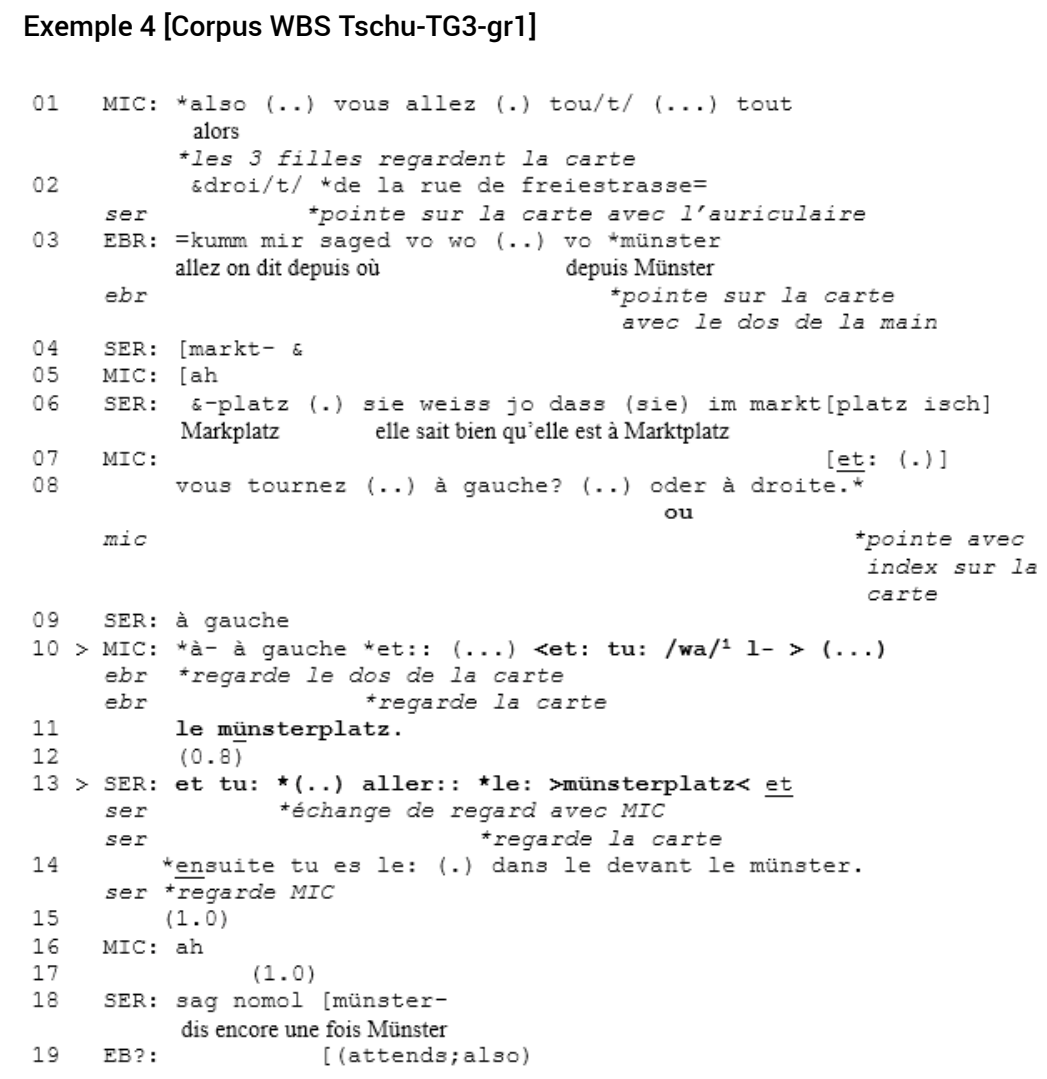

23 Après avoir rencontré de nombreuses difficultés, ainsi qu'en témoignent les nombreuses pauses et allongements vocaliques notamment, Michelle arrive à la fin de sa description de l'itinéraire aux lignes 10-11 avec la mention du point d'arrivée : le Münsterplatz. Après une pause inter-tours de 0.8 seconde, Serife intervient en reprenant un segment discursif large, qui correspond à la dernière proposition du tour de Michelle, et remplace la forme standard 'tu /wa/' par une autre 'tu aller'. Ce faisant, Serife rend compte qu'elle traite la forme 'tu /wa/' comme étant problématique, alors même que Michelle n'avait pas manifesté d'attention particulière envers cet élément linguistique.

24 A l'instar de l'exemple précédent, la reprise est immédiatement suivie d'un connecteur ('et ensuite', l.13-14) projetant un développement discursif à venir. De plus, le débit accéléré sur 'münsterplatz', élément du segment-repris (l. 13) permet de passer 'rapidement' à la suite, sans laisser la possibilité à quiconque de prendre la parole à ce moment-là. Il est à noter que la transition entre le segment-repris et le développement discursif ('>münsterplatz< et ensuite tu es', 1. 13-14) est accomplie de manière fluide, ce qui contraste avec les hésitations (pauses et allongements vocaliques) qui jalonnent le tour de parole de Serife. Ainsi, cette fluidité sur la frontière entre la reprise et l'expansion discursive est également une ressource grâce à laquelle Serife signale que son intervention va au-delà de la correction linguistique. Michelle ne ratifie que 
minimalement l'énoncé-reformulateur de Serife ; la correction linguistique n'est donc pas focalisée par les participantes. L'échange continue avec la poursuite de la tâche.

En somme, ces deux exemples présentent un formatage interactionnel semblable de la reprise corrective :

1. L'objet de la correction n'a pas été focalisé par le premier locuteur.

2. La correction n'est ni rendue saillante par le locuteur B, ni reconnue expressément par le locuteur A par la suite.

3. La reprise est suivie d'un développement discursif; ces deux parties s'enchaînent de manière fluide (sur le plan syntaxique et prosodique).

Ces différents éléments participent à l'accomplissement d'une correction implicite, voire atténuée. Le fait de ne pas attirer l'attention explicitement sur l'activité corrective permet de mener le projet communicatif à bien, sans devoir l'interrompre. Ce type de correction s'apparente ainsi à ce qui a été appelé ailleurs 'embedded correction' ou correction 'incorporée' (Jefferson, 1987 ; voir aussi Brouwer et al. 2004 ; Fasel Lauzon \& Pekarek Doehler, 2013; Svennenig, 2004) où les participants d'un échange traitent les problèmes linguistiques en même temps que sont accomplies d'autres activités (p.ex. répondre à une question, poursuivre une description, etc.), par opposition à des 'exposed corrections' (Jefferson, 1987) ou des corrections réalisées ouvertement. A propos des corrections incorporées, Jefferson (1987 : 95) précise : « correction occurs, but is not what is being done, interactionally. » Autrement dit, la correction n'est pas mise au centre de l'attention des participants, lesquels s'affairent avec d'autres activités communicatives. Dans cette optique, la reprise se prête particulièrement bien à une correction implicite, puisqu'elle permet simplement de remplacer l'élément problématique au sein d'un projet communicatif plus large sans faire intervenir une focalisation explicite sur cet objet.

\section{Reprise et gestion interactionnelle de la tâche}

Cette deuxième section analytique se préoccupe des enjeux interactionnels impliqués au moment de la reprise corrective. Les analyses montreront comment l'activité corrective est étroitement imbriquée dans la gestion de l'attention mutuelle, de l'accomplissement conjoint de la tâche et du maintien des rapports interpersonnels.

Reprenons les deux exemples analysés précédemment. Dans l'exemple 5 (cf. exemple 3), Lorena et Michelle établissent conjointement une liste d'objets à acheter pour la fête : 
Exemple 5
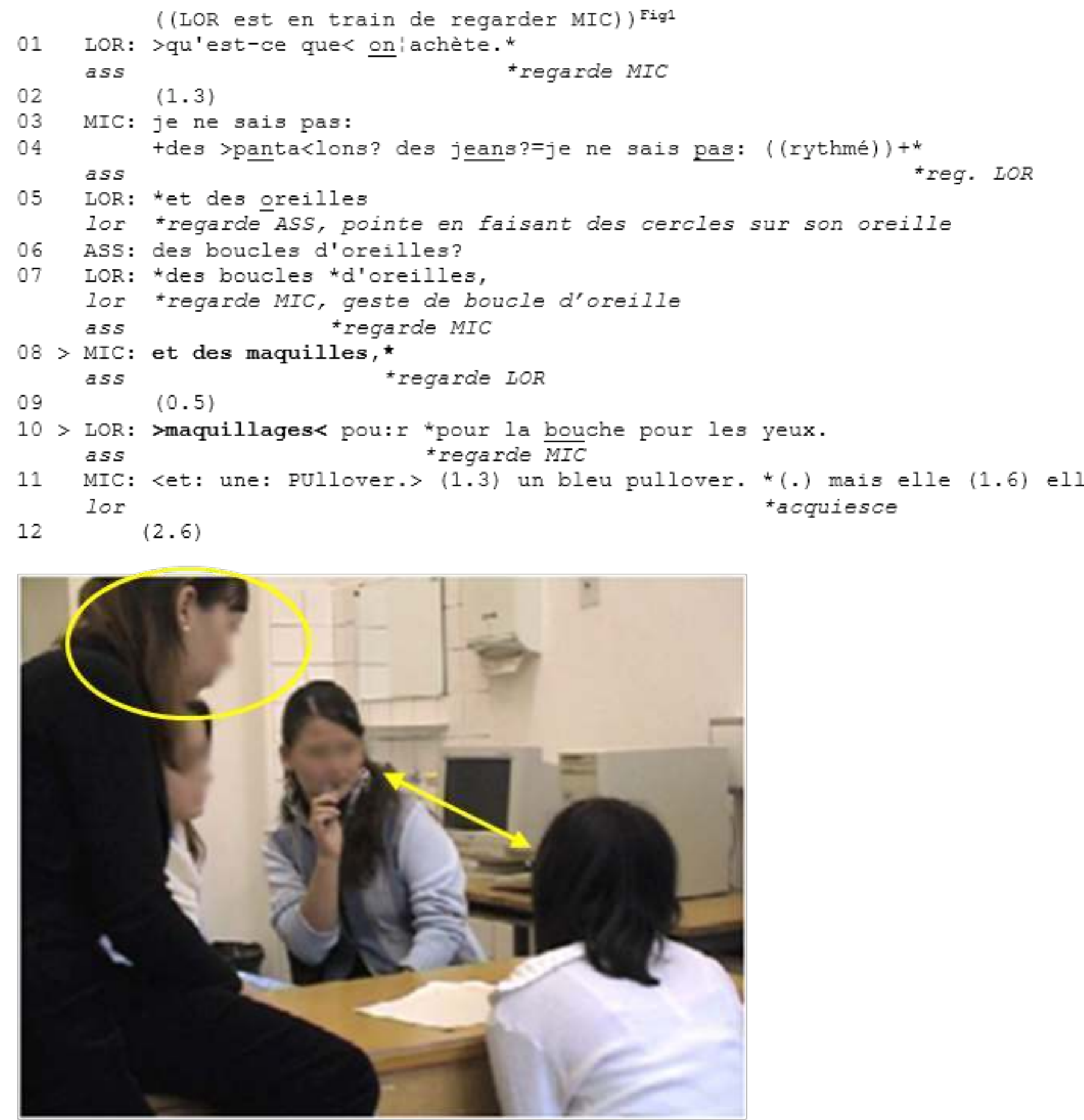

Fig. 1. Lorena et Michelle se regardent.

29 Si on regarde l'élaboration collective de la liste, on constate que Lorena et Michelle proposent chacune à leur tour un candidat à l'achat. Ces propositions ne font pas l'objet d'un commentaire ou d'une ratification explicite par la co-participante, mais semblent être acceptées tacitement par le biais d'un enchaînement avec une nouvelle proposition : 'des pantalons' (1.4), 'et des boucles d'oreilles' (1. 5), 'et des maquilles' (1. 8), 'et une pullover' (1. 11). Le seul candidat faisant l'objet d'une réaction de la co-participante est 
précisément la proposition de Michelle contenant la forme non-standard 'et des maquilles'. Alors que cette forme non-standard n'est pas traitée comme problématique par la locutrice elle-même qui s'oriente au contraire vers la poursuite de la liste (voir analyse plus haut), elle semble poser problème à sa camarade. En effet, au lieu de fournir un nouveau candidat à la suite de Michelle, Lorena laisse s'écouler une pause de 0.5 seconde avant de prendre la parole - qui consiste en une correction du tour de Michelle alors qu'elle est traitée par celle-ci comme étant la prochaine locutrice (cf. regard, 1.8).

La forme lexicale 'maquilles', en plus de faire l'objet d'une correction dans le tour suivant fait également l'objet d'une description détaillée ('pour la bouche pour les yeux', 1. 10). Ce faisant, Lorena démontre doublement qu'elle a bien compris l'élément lexical dont la première mention par Michelle est traitée comme problématique. Du fait que la correction est intégrée dans une expansion discursive, Lorena reconnaît publiquement l'énoncé-source comme étant orienté vers les contenus et la progression de la tâche et non pas vers les formes linguistiques. La reprise corrective et l'expansion permettent donc de maintenir la progressivité du discours (Stivers \& Robinson, 2006), de façon à mener la construction de la liste à son terme et ainsi clore cette partie de la tâche ; car un traitement explicite du problème linguistique aurait engendré une suspension momentanée de l'activité descriptive. De ce fait, la reprise corrective permet de préserver l'intersubjectivité entre les participants - nécessaire à la gestion conjointe de l'interaction et de la tâche - là où elle aurait pu potentiellement être mise en cause.

31 Il est à noter également que cet échange entre Lorena et Michelle se déroule face à d'autres participantes: Olivia, qui participe au travail en groupe, et l'assistante de l'enseignant. La présence de cette dernière n'est pas sans conséquence. En effet, cet échange est en quelque sorte mis en scène de façon à montrer à l'assistante que le groupe a bien fait son travail jusque-là, ainsi qu'en témoignent les regards exclusifs entre Lorena et Michelle dans cet extrait (cf. figure 1), qui ne s'orientent vers l'assistante que pour faire appel à son expertise linguistique lorsqu'un problème se pose (1. 5). Ainsi, face à un évaluateur potentiel, Lorena semble mettre la priorité sur une dynamique collaborative et une relation d'égalité entre les membres du groupe; car, une correction explicite des propos de sa camarade reviendrait à lui attribuer indirectement un manque de compétence linguistique. A ce propos, Jefferson (1987) souligne en effet que l'enjeu des corrections explicites n'est pas seulement d'exprimer des choses correctement, mais de pointer des lacunes ou des incompétences chez son interlocuteur.

Dans l'exemple 6 (cf. exemple 4), trois filles sont en train d'élaborer conjointement un itinéraire : 


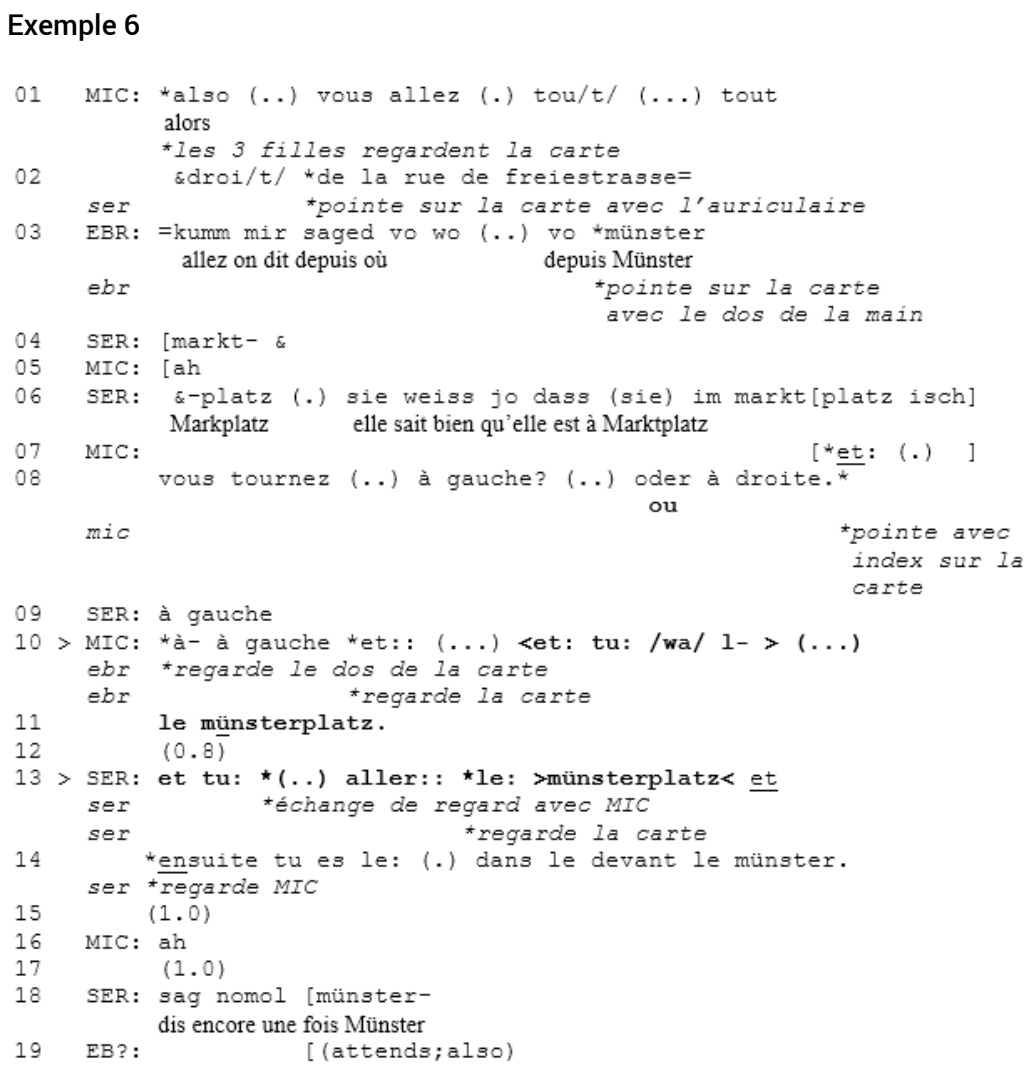

33 La reprise corrective que produit Serife à la ligne 13 des propos de Michelle ne concerne pas uniquement une modification au niveau de la forme (et notamment, la reformulation de la forme verbale /wa/ en 'aller'), mais aussi des contenus informationnels. En effet, Serife semble traiter la description de Michelle, et notamment l'étape finale de l'itinéraire (c'est-à-dire l'arrivée au point-cible) comme n'étant pas suffisamment précise. Ainsi, sa reformulation de l'étape finale de l'itinéraire est suivie d'une expansion discursive apportant un complément d'information à l'identification du point d'arrivée : 'et ensuite tu es devant le münster' (1. 14). Ce faisant, elle effectue non seulement une réparation des contenus informationnels mais réactualise sur le plan actionnel la clôture de la séquence descriptive en en proposant une alternative plus détaillée. D'ailleurs, dans la suite de la tâche (au-delà de l'extrait ci-présent), cette information additionnelle sera rappelée à plusieurs reprises lors des différentes reformulations de l'itinéraire. Cela montre à quel point cet ajout d'information concernant l'identification du point d'arrivée est pertinent pour les participantes.

Serife se réapproprie donc une activité qui était menée sous la responsabilité d'une camarade et se positionne comme gestionnaire de la tâche et comme experte, qui évalue et ratifie l'adéquation des productions de ses camarades à la fois au niveau linguistique et informationnel (cf. aussi l'hétéro-correction, 1. 4-6 et la réponse à l'hésitation de Michelle, 1. 9), soutient ses camarades (cf. geste de pointage sur la carte, 1. 2), et décide de refaire encore une fois la description (1. 18). Il est à noter cependant qu'au moment de sa prise de tour, Serife jette un rapide coup d'œil à Michelle (1. 13), semblant chercher l'attention, voire l'accord, de Michelle pour effectuer la réparation, avant de s'orienter à nouveau vers la carte et poursuivre la tâche. Dans cet extrait, la reprise corrective permet de négocier les contenus informationnels. Mais au contraire de l'exemple 5 , où la reprise corrective instaurait une relation d'égalité entre les membres du groupe, ici c'est un 
rapport de force qui est établi entre les participantes. En fait, l'activité même de reformulation confère à la personne qui reformule une position dominante par rapport à celle dont les propos sont justement reformulés.

Regardons à présent un exemple provenant d'un autre groupe travaillant également sur la description d'itinéraire. Avec l'extrait suivant, le groupe s'apprête à expliquer comment aller de la Messeplatz à Sankt Jakob. L'explication est initiée par Lorena (qui avait été désignée plus tôt en tant que responsable de cette partie de la tâche) :

\section{Exemple 7 [Corpus WBS Tschu-TG3-gr3]}

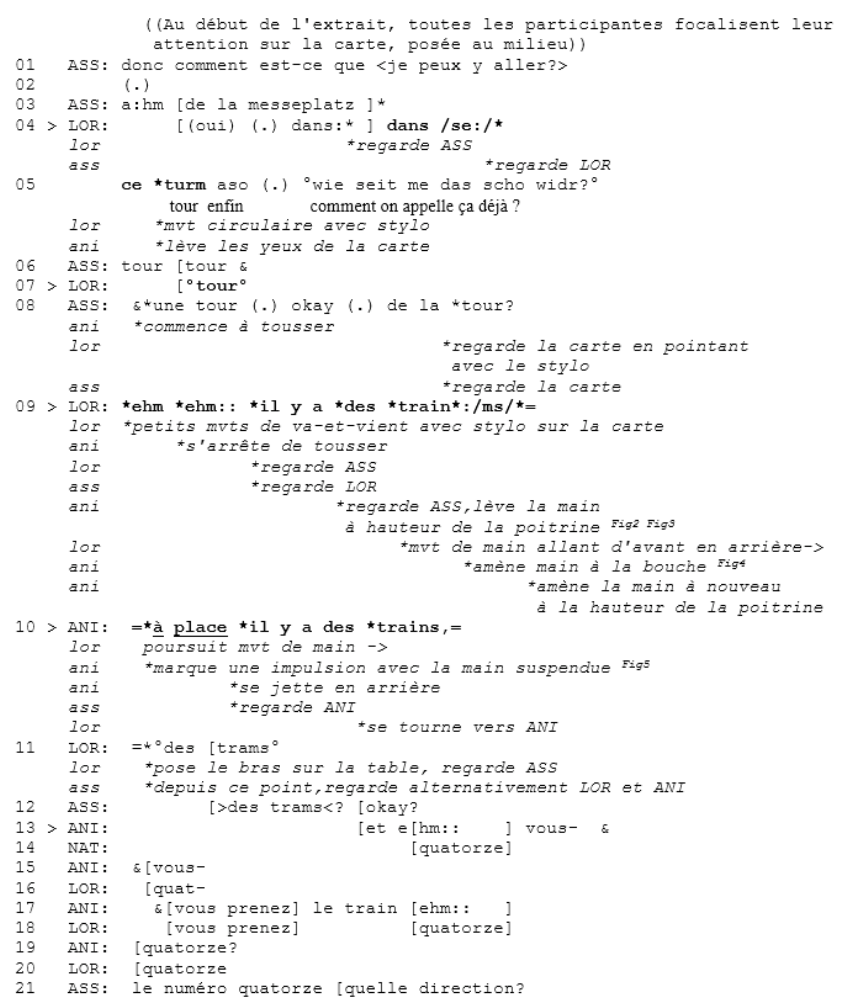





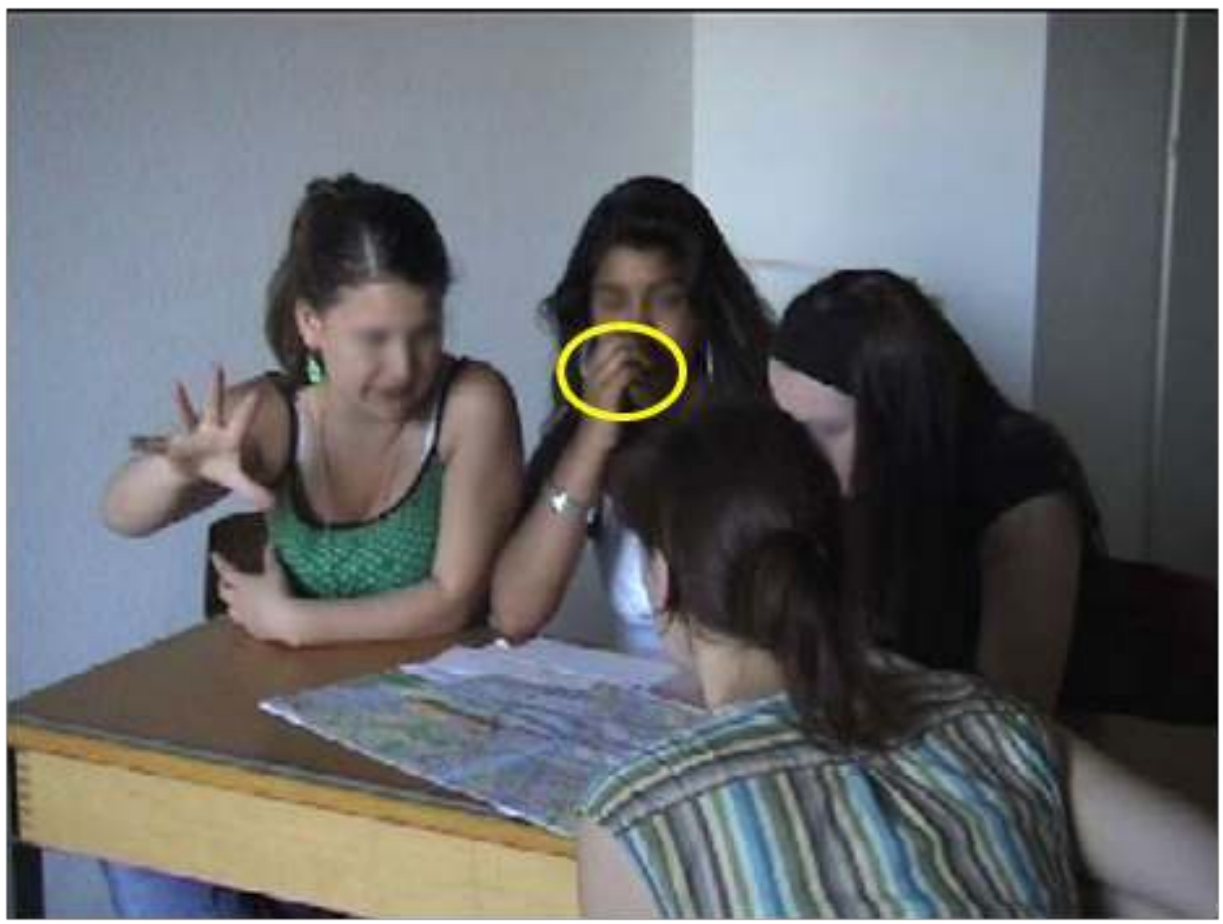

Fig.4. Anila amène sa main vers la bouche.

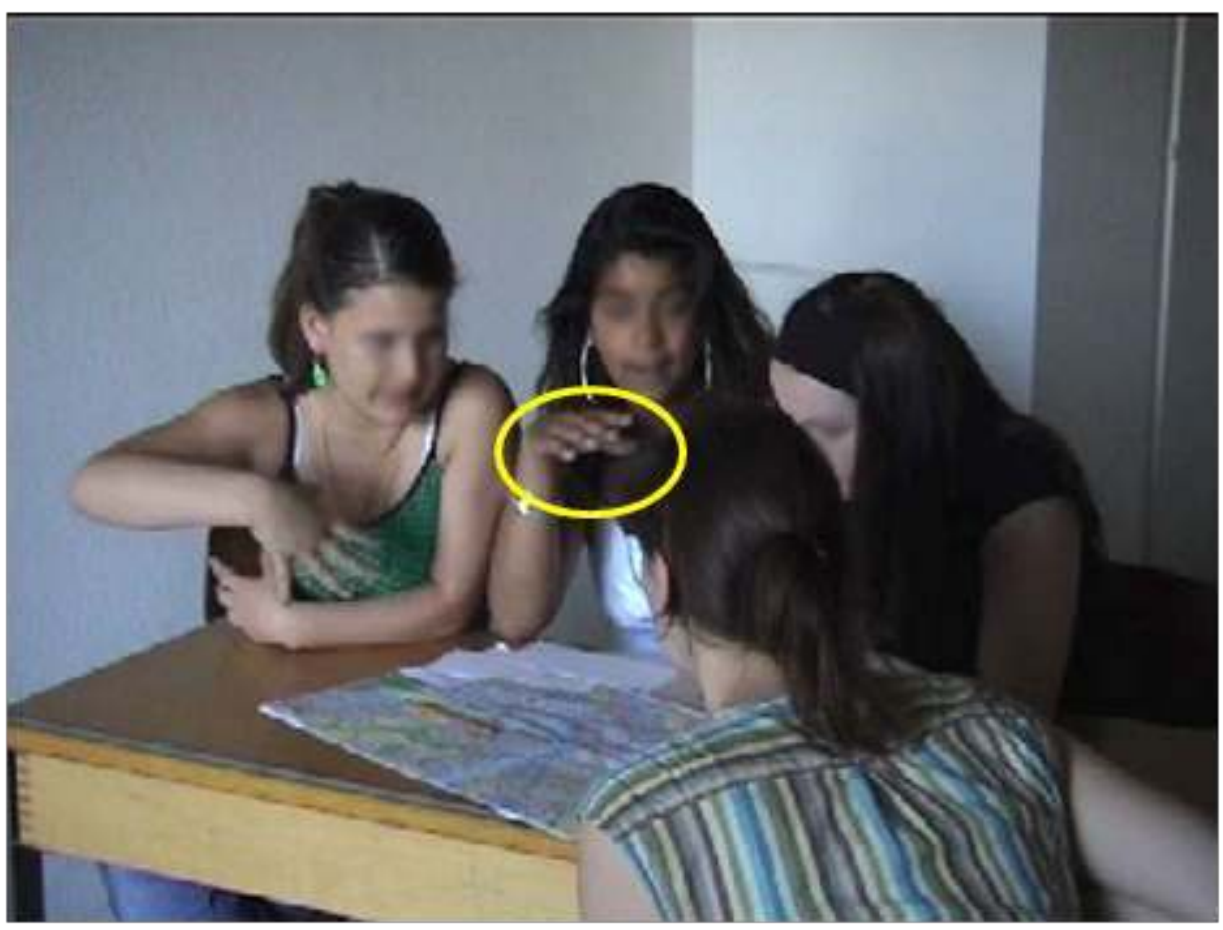

Fig.5. Anila fait un mouvement avec sa main.

Dès la ligne 4, Lorena initie la description de l'itinéraire, l'assistante la regarde alors. Cette description est cependant produite avec des hésitations : recherche lexicale (1. 4-5), étayage de l'assistante (1. 6-8), d'autres marqueurs d'hésitations suivent (1. 9). Elle finit par poursuivre sa description, cependant hésite entre deux éléments lexicaux ('trains' et 'trams'), ce qui se traduit par une forme hybride 'trainms' produite avec allongement syllabique. Anila enchaîne sur cette forme lexicale hésitante produite par Lorena, sans 
laisser la possibilité à quiconque de prendre la parole à ce moment-là. Sa prise de tour consiste en une reprise corrective de la description initiée par Lorena, reformulant le complément de lieu en 'à place', et choisissant l'alternative 'trains' au lieu de 'trainms'. (Il est à noter que la formulation d'Anila n'est pas plus acceptable sur le plan normatif que celle de Lorena.) A la différence des exemples vus jusqu'ici, la reprise porte dans cet exemple 7 sur un segment discursif distribué à travers plusieurs tours de parole. De plus, le parallélisme entre le segment-source et la reprise est ici moins étroit que pour les exemples précédents; il se manifeste au niveau des catégories syntaxiques avec le remplacement du complément de lieu 'dans ce tour' par 'à place'. Finalement, les hésitations et les erreurs linguistiques que Lorena produit au cours de sa description initiale semblent constituer une occasion pour Anila de s'insérer dans l'échange qui se déroule exclusivement entre Lorena et l'assistante au début de l'extrait (cf. regard mutuel, 1.4). La reprise corrective apparait comme un moyen légitime d'intervenir - et donc de s'approprier la parole - après le tour de Lorena.

En plus de la reprise corrective, Anila mobilise également des ressources prosodiques et visuelles pour s'approprier le tour de parole. D'une part, l'entrée dans le tour d'Anila est marquée sur le plan prosodique par une accentuation initiale qui permet de signaler de manière audible qu'elle a pris la parole. D'autre part, Anila procède à un réarrangement corporel, avant même sa prise de tour, qui lui permet d'entrer dans le champ de vision de l'assistante. Elle adopte alors une position d'élocution: elle se redresse corporellement (figures 2 et 3), pose son coude sur la table (figure 3), puis amène sa main à la bouche (figure 4). Par la suite, elle gesticulera avec cette même main, à la hauteur du regard de l'assistante (figure 5). Ces différentes ressources (verbales, prosodiques et corporellesgestuelles) lui permettent d'attirer l'attention de l'assistante afin d'être ratifiée comme locutrice légitime et d'intégrer un cadre de participation dont elle était exclue jusqu'alors (cf. les regards de Lorena et de l'assistante, 1. 10).

Malgré les réactions de Lorena et de l'assistante, Anila poursuit son tour de parole avec une expansion (1.13). Dès ce moment-là, Anila est ratifiée en tant que locutrice et aussi en tant que co-responsable de la description de l'itinéraire, puisque l'assistante regardera désormais les deux élèves Lorena et Anila en alternance (l. 11). Les deux participants se trouvent alors en compétition pour la parole: chacune cherche à obtenir et garder l'attention de l'assistante, ainsi qu'à mener la description à son terme. Cette compétition se traduit non seulement au niveau du discours, avec les nombreux chevauchements (l. $13,16,18,20)$; mais également au niveau physique, les deux filles étant épaule à épaule dans une position corporelle semblable (cf. figure 4 et 5).

En somme, il apparait dans cet extrait que l'hétéro-reprise en début de tour et dans un contexte de compétition pour la parole, constitue un procédé discursif 'économique', c'est-à-dire rapidement mobilisable, dans la mesure où le matériel linguistique est déjà disponible dans le discours et peut être réutilisé facilement, d'autant plus que sur le plan informationnel la reprise n'est que très peu pertinente. Ainsi, Anila peut rapidement accéder au terrain de la parole, sans encore avoir planifié la suite de sa contribution: en effet, elle ne produit du nouveau matériel linguistique qu'à partir de la ligne 14. D'autre part, du fait que sa camarade rencontre des difficultés d'ordre linguistique, l'activité corrective est tout à fait légitime dans ce cadre et permet à Anila de proposer une version alternative de la description. Dans ce contexte, la reprise corrective joue donc un rôle de dispositif d'entrée dans le tour, qui inscrit le tour de parole ainsi initié de manière pertinente dans la continuité avec les tours précédents. Sur le plan des rapports 
interpersonnels au sein du groupe, Anila procède de manière subtile à un remaniement des cadres de participation de façon à pouvoir se positionner en locutrice légitime de l'activité.

\section{Discussion et conclusion}

Les analyses ont montré un formatage récurrent de la reprise impliquée dans une activité de correction, laquelle n'est pas focalisée par les participants. En effet, de manière systématique, la modification d'un élément linguistique du tour antérieur n'est ni mis en évidence par le locuteur effectuant la reprise, ni ratifié subséquemment par l'auteur de l'énoncé-source. Par ailleurs, ces reprises correctives sont immédiatement suivies d'une expansion discursive. Ces deux observations indiquent que les participants ne s'engagent pas à proprement parler dans une activité de correction, mais l'intègrent subtilement dans la poursuite du projet communicatif initial. La correction est ainsi 'camouflée', ce qui permet d'éviter une interruption du flux communicatif (Jefferson, 1987).

41 De manière générale, ces résultats convergent avec des observations similaires sur des hétéro-reprises de type non-correctif (cf. Berger, 2008; Pochon-Berger, 2010). Ces fonctionnements similaires font apparaitre un pattern interactionnel récurrent incluant à la fois des aspects linguistiques et séquentiels : des hétéro-reprises immédiates en position initiale du tour et enchaînant avec une expansion discursive (cf. section 3). Il s'agit là d'un pattern interactionnel productif, puisque les participants y ont recours pour faire face à toutes sortes d'enjeux pratiques: établir une cohérence inter-tours, garantir la progressivité du discours, négocier les formes et les contenus, obtenir l'attention des coparticipants et reconfigurer les cadres de participation. Par ailleurs, elle intervient également dans la gestion des relations interpersonnelles indispensables à un travail de groupe, par le maintien de l'intersubjectivité, l'établissement de rôles et de positions au sein d'un groupe et la recherche de consensus et de coopération entre les participants.

Les recherches antérieures sur la reprise et la reformulation verbales ont identifié une panoplie de fonctions pour ce phénomène discursif. De la même façon, les trois extraits présentés ici font apparaitre une image complexe de fonctions communicatives étroitement imbriquées à travers un même procédé discursif. La diversité des fonctions identifiées démontre le caractère hautement situé de la reprise (corrective ou non), laquelle prend son sens dans le contexte spécifique où elle est mise en œuvre. Elle constitue dès lors une ressource flexible, adaptative, pouvant être mobilisée à toutes fins pratiques. La participation à des interactions verbales suppose la gestion de nombreux aspects: structuration des formes linguistiques, organisation des contenus informationnels et coordination des activités mutuelles. Dans cette optique, en tant que procédé discursif et séquentiel, la reprise permet justement la gestion simultanée de ces différents niveaux.

Sur le plan méthodologique, ces résultats invitent à repenser les critères sur lesquels s'établissent les catégories d'analyse. Au lieu d'appliquer des catégories descriptives préexistantes aux données sur la base de critères formels, c'est la manière dont les participants eux-mêmes s'orientent vers la reprise qui est privilégiée. Dans cette perspective, ce sont les propriétés séquentielles de la reprise, témoignant de la construction moment après moment des cours d'action, qui sont pertinentes pour l'analyse. Ces propriétés séquentielles permettent par exemple d'intégrer l'occurrence 'à place il y a des trains' (cf. exemple 7) dans la collection, même si la reprise du complément de lieu témoigne d'un certain degré d'éloignement formel avec le segment-source. Elles 
permettent aussi de prendre en compte un cas de reprise, dont l'énoncé-source est distribué sur plusieurs tours de parole (cf. exemple 7). L'enjeu pour le chercheur n'est dès lors plus de dresser un inventaire des formes et des fonctions de la reprise ou de la reformulation, mais de voir comment ce procédé, en tant qu'activité de retour sur le dire d'autrui, est utilisé par les participants dans un contexte donné comme ressource pour gérer les circonstances uniques à un événement.

Enfin, pour ce qui est de l'activité corrective réalisée au moyen de la reprise, les résultats soulignent l'importance de prendre en compte les détails séquentiels, prosodiques et nonverbaux dans l'examen des activités de correction. C'est seulement au travers de ces détails que peut être saisie la manière dont les participants eux-mêmes se positionnent par rapport à l'activité de correction et s'orientent ou non vers un problème linguistique devant être résolu. Cela implique de repenser des objets d'étude classiques en acquisition des langues tels que la notion d'erreur, l'attention portée aux formes ou encore l'auto-/ hétéro-correction dans la perspective des participants eux-mêmes (cf. Fasel Lauzon \& Pekarek Doehler, 2013).

Aussi les analyses montrent-elles que le potentiel acquisitionnel des reprises correctives ne réside pas tant dans le travail métalinguistique qui est accompli à travers elles, mais plutôt dans les opportunités de participation qui sont mises en œuvre par ce biais. Si l'on considère que l'apprentissage des langues secondes se fonde sur la participation régulière aux activités d'une communauté donnée (cf. Hall, 1993; Mondada \& Pekarek Doehler, 2001), alors la reprise y joue un rôle clé. En tant que ressource pour interagir, la reprise constitue ainsi une trace d'une compétence d'interaction (cf. Hall \& Pekarek Doehler, 2011), certes en développement, mais permettant certaines formes de participation à un niveau de français langue seconde peu avancé.

\section{BIBLIOGRAPHIE}

BERGER, E., (2008), « La reprise comme ressource interactionnelle », TRANEL (Travaux Neuchâtelois de Linguistique), 48, 43-61.

BROUWER, C.E., (2004), « Doing pronunciation : A sequence of novice-expert interaction », dans Gardner, R. \& Wagner, J. (eds.), Second language conversations. London, Continuum, p.93-113.

BROUWER, C.E., RASMUSSEN, G. \& WAGNER, J., (2004), « Embedded corrections in second language talk », dans Gardner, R. \& Wagner, J. (eds.), Second language conversations. London, Continuum, p.75-92.

De PIETRO, J.-F., MATTHEY, M. \& PY, B., (1989), « Acquisition et contrat didactique : les séquences potentiellement acquisitionnelles dans la conversation exolingue », dans Weil, D. \& Fugier, H. (éds.), Actes du $3^{e}$ colloque régional de linguistique. Strasbourg, Université des sciences humaines et Université Louis Pasteur, p.99-124.

FARACO, M., (2002), « Répétition, acquisition et gestion de l'interaction sociale en classe de L2 », Acquisition et Interaction en Langue Etrangère, 16, 97-120. 
FASEL LAUZON, V. \& PEKAREK DOEHLER, S, (2013), « Focus on form as an interactional accomplishment ", International Review of Applied Linguistics, 51 (4), 323-351.

GÜLICH, E. \& KOTSCHI, T., (1983), «Les marqueurs de la reformulation paraphrastique », Cahiers de Linguistique française, 5, 305-351.

HALL, J.K., (1993), « The role of oral practices in the accomplishment of our everyday lives : the sociocultural dimension of interaction with implications for the learning of another language ", Applied Linguistics, 14 (2), 145-166.

HALL, J.K. \& PEKAREK DOEHLER, S, (2011), « Introduction : Interactional Competence and Developement », dans Hall, J.K., Hellemann, J. \& Pekarek Doehler, S. (eds.), L2 Interactional Competence and Development. Clevedon, Multilingual Matters, p.1-15.

HELLERMANN, J., (2011), « Members' methods, members' competencies : looking for evidence of language learning in longitudinal investigations of other-initiated repair », dans Hall, J.K., Hellermann, J. \& Pekarek Doehler, S. (eds.), L2 Interactional Competence, Clevedon, Multilingual Matters, p.147-172.

HOSODA, Y., (2000), « Other-repair in Japanese conversations between nonnative and native speakers », Issues in Applied Linguistics, 11 (1), 39-63.

JEFFERSON, G., (1987), « On exposed and embedded correction in conversation », dans Button, G. \& Lee, J.R.E. (eds.), Talk and social organisation. Clevedon, Multilingual Matters, p.86-100.

KRAFFT, U. \& DAUSENDSCHÖN-GAY, U., (1993), « La séquence analytique », Bulletin CILA [VALSASLA], 57, 137-158.

KURHILA, S., (2006), Second language interaction, Amsterdam, John Benjamin.

LYSTER, R. ,(1998), « Recasts, repetition, and ambiguity in L2 classroom discourse », Studies in Second Language, 20, 51-81.

LYSTER, R. \& RANTA, L., (1997), « Corrective feedback and learner uptake : negotiation of form in communicative classrooms », Studies in Second Language Acquisition, 19, 37-61.

MONDADA, L. \& PEKAREK DOEHLER, S., (2001), « Interactions acquisitionnelles en contexte. Perspectives théoriques et enjeux didactiques», Le français dans le monde. Numéro spécial (juillet 2001), 107-137.

PALLOTTI, G., (2002), « Borrowing words », dans Leather, J. \& van Dam, J. (eds.), The ecology of language acquisition, Amsterdam, Kluwer Academic Publishers, p.183-202.

PEKAREK DOEHLER, S. \& POCHON-BERGER, E., (2010), « La reformulation comme technique de gestion du désaccord : le "format tying" dans les interactions en classe de langue ", dans Rabatel, A. (éd.), Les reformulations dans des situations pluri-sémiotiques, en contexte didactique. Besançon, Presses Universitaires de Franche-Comté, p.117-134.

POCHON-BERGER, E., (2010), La compétence d'interaction en L2 : gestion de la cohérence interactive par des apprenants $d u$ français. Thèse de doctorat, Université de Neuchâtel, 2010. [disponible en ligne sur http ://doc.rero.ch/lm.php ?url=1000,40,4,20100929144628-EC/00002167.pdf ]

RABATEL, A., (2010), « Pour une approche intégrée des reformulations pluri-sémiotiques en contexte de formation : apprendre en reformulant et en resémiotisant documents iconiques, gestes et actions ", dans Rabatel, A. (ed.), Les reformulations pluri-sémiotiques en contexte de formation, Besançon, Presses universitaires de Franche-Comté, p.7-24.

SALZAR ORVIG, A., (2000), « La reprise aux sources de la construction discursive », Langages, 140, 68-9. 
SVENNENIG, J., (2004), « Other-repetition as display of hearing, understanding and emotional stance », Discourse Studies, 6, 489-516.

STIVERS, T. \& ROBINSON, J., (2006), « A preference for progressivity in interaction », Language in society, 35, 367-392.

TANNEN, D., (1989), Talking voices : repetition, dialogue and imagery in conversational discourse, Cambridge, Cambridge University Press.

VASSEUR, M.-T., (2005), Rencontres de langues. Question(s) d'interaction. Paris, Editions Didier.

VION, R., (1992), La communication verbale. Analyse des interactions. Paris, Hachette.

VION, R. \& MITTNER, M., (1986), « Activités de reprise et gestion des interactions en communication exolingue », Langages, 84, 25-42.

\section{ANNEXES}

\section{Conventions de transcription}

[ : début de chevauchement de tours de parole

]: fin de chevauchement de tours de parole

$=$ : enchaînement rapide entre deux tours de parole ou deux mots à l'intérieur d'un tour $\&$ : continuation d'un tour de parole

(.) (..) (...) : pauses en-dessous de 1.0 seconde

(2.5) : pause au-dessus de 1.0 (avec indication de la durée en seconde)

$\mathrm{j}$ - : troncation du mot

de: : allongement syllabique

oui ?intonation finale montante

non. : intonation finale descendante

maquillages, : intonation finale continuative

hôtel : accentuation d'une syllabe

VRAI : augmentation de volume

${ }^{\circ}$ oui' ${ }^{\circ}$ baisse de volume

>un peu< : accélération du débit

$<$ un peu : ralentissement du débit

.h : inspiration

h. : expiration

(non) : transcription d'un mot incertain

(xx) : mot incomprénsible

(je; j'ai) : transcription incertaine entre deux alternatives

((rires)) : commentaire du transcripteur; si le commentaire porte sur un segment délimité, alors la délimitation est indiquée par +

* : balise indiquant le début d'un comportement non-verbal décrit à la ligne concernant la multimodalité

trad : traduction 


\section{RÉSUMÉS}

La présente étude se penche sur les reprises correctives au sein d'interactions scolaires en classe de français langue étrangère. Sur la base d'un corpus d'interactions en petits groupes, une analyse qualitative fine a été menée sur une collection d'hétéro-reprises séquentiellement immédiates. Les cas observés montrent que ces reprises, bien qu'elles effectuent une modification d'un élément linguistique dans le tour antérieur, ne focalisent pas sur l'activité de correction en soi. En effet, bien au-delà du travail métalinguistique, l'hétéro-reprise est utilisée comme ressource interactionnelle, notamment pour la gestion de la prise de tour et le maintien de la progressivité de l'interaction.

This study investigates corrective repetitions in French FL classroom interactions. Drawing on a corpus of small group work interactions, we carried out a qualitative analysis of a collection of sequentially immediate other-repetitions. The observed cases show that these other-repetitions do not focus on the activity of correcting in itself, although a linguistic item of the prior turn is being modified. Indeed, beyond the mere metalinguistic work, the other-repetition is used by participants as an interactional resource for getting a turn-at-talk and for securing the progressivity of talk-in-interaction.

\section{INDEX}

Mots-clés : hétéro-reprise ; prise de tour ; progressivité du discours ; classe de langue ; travail en groupe; français langue seconde

Keywords : other-repetitions; turn-at-talk; progressivity of discourse; language class; group work; French as a foreign language

\section{AUTEUR}

\section{EVELYNE BERGER}

Université de Helsinki 